\title{
On periodic solutions and their modulations for the Manakov system
}

\author{
A M Kamchatnov \\ Institute of Spectroscopy, Russian Academy of Sciences, Moscow, Troitsk, 142190, \\ Russia \\ E-mail: kamch@isan.troitsk.ru
}

\begin{abstract}
Periodic solutions of the Manakov system are studied with the aim to establish links between recent results of Wright (Physica D 2013264 1) and Kamchatnov (EPL 2013103 60003), where the one-phase solutions have been obtained by different methods and with different parameterizations. Two types of waves, which in the context of dynamics of two-component Bose-Einstein condensates can be called density and polarization waves, are determined as particular solutions of the Manakov system. Whitham equations describing modulations of these two types of waves are obtained.
\end{abstract}

PACS numbers: 02.30.Ik, 03.75.Mn, 42.65.Tg 


\section{Introduction}

Periodic solutions of nonlinear wave equations play an important role in the theory of dispersive shock waves and in other their applications to physics. If a nonlinear wave equation is completely integrable by the inverse scattering transform approach, then periodic and quasi-periodic solutions of this equation can be obtained by the powerful finite-gap integration method (see, e.g., [1] and references therein). In particular, the complete integrability of a very important for applications nonlinear Schrödinger (NLS) equation was discovered in [2] and periodic solutions of this equation were obtained by the finite-gap method in [3, 4, 5]. The solutions found in this way do not have convenient enough form because an additional constraint is imposed upon the variables used in the method. This constraint singles out the real solutions from the general finite-gap formulae and it is difficult to satisfy this additional condition in the multiphase quasi-periodic case. As was shown in [6], the additional constraint can be resolved explicitly to determine the loci of the auxiliary spectral variables used in the finite-gap method in the most important for applications case of the single-phase periodic solutions of the NLS equation and later this technique was extended to many other single-phase solutions of integrable equations [7, 8] which belong to the Ablowitz-Kaup-Newell-Segur (AKNS) scheme [9].

There are integrable equations which do not belong to the AKNS scheme and the method of [6] cannot be applied to them directly. One of the most important such equations is the Manakov system [10] representing two coupled NLS equations and having applications to nonlinear optics and Bose-Einstein condensates (BEC) physics. Some particular solutions of the Manakov system have been studied in [11, 12, 13] by introducing a special ansatz for the form of the solution so that the problem is reduced to the AKNS-type scheme. The finite-gap integration method was applied to the Manakov system by Shin [14], however, the reality conditions were not resolved in this paper. Wright has recently succeeded [15] in extension of the method of [6] to the Manakov system so that the reality conditions are completely characterized and the general formulae for the single-phase solution are obtained. It is also shown that the Manakov soliton solution can be recovered in the corresponding limit. A similar solution was obtained in [16] by a direct method based on introduction of new dependent variables whose form was motivated by physical interpretation of the Manakov system as the Gross-Pitaevskii equations describing dynamics of the two-component BECs. In this context it is natural to distinguish the in-phase and counter-phase motions of the components, and the new variables permit one to describe separately these two modes of BEC dynamics. As a result, the periodic solutions corresponding to the density (inphase) and the polarization (counter-phase) waves as well as to their combination were obtained. Such a distinction between two kinds of motion has not been considered in [15] and the aim of this paper is to show that the solutions studied in detail in [16] can be obtained in framework of the scheme developed by Wright.

The advantage of the finite-gap integration method is that the periodic solutions are 
parameterized by the parameters which play the role of the Riemann invariants in the Whitham theory for slowly modulated wavetrains [17]. This property of the method was first demonstrated for the Korteweg-de Vries equation in [18] and it was extended to the NLS equation in [19, 20]. A simple method of derivation of the Whitham equations for single-phase wavetrains in framework of the finite-gap approach was suggested in [21, 22 . and it was applied to many integrable equations in [7, 8]. Wright supposed that similar parameters (namely, branch points of the trigonal spectral curve which in the Manakov case replaces the elliptic spectral curve appearing in the NLS case) play the same role in the Whitham theory for the Manakov system and confirmed this supposition by a nontrivial example of the dispersionless limit of this system [23. In Section 3 of the present paper we consider the Whitham equations for modulations of purely density waves and show that a simple extension of the method developed in [21, 22] leads to equations coinciding with those [19, 20] for the NLS case. This result is physically natural - for synchronized in-phase motion of two components difference between them disappears and their dynamics reduces to the one-component one. However, the modulations of the polarization waves are not described by the same parameters (i.e., by the branch points of the spectral curve) and the corresponding Whitham equations for a purely polarization wave are obtained by the direct Whitham method of averaging of the conservation laws. These Whitham equations can be transformed to the Riemann diagonal form and they can be integrated the standard method of characteristics. The results obtained for these two particular types of the periodic solutions of the Manakov system can be applied to relevant problems of BEC dynamics.

\section{Periodic solutions}

In this section we describe briefly the main results of Refs. [15] and [16] with the goal to establish links between these two forms of periodic solutions of the Manakov system. We shall consider the Manakov equations in the form

$$
i \partial_{t} \psi_{ \pm}+\frac{1}{2} \partial_{x x}^{2} \psi_{ \pm}-\left(\left|\psi_{ \pm}\right|^{2}+\left|\psi_{\mp}\right|^{2}\right) \psi_{ \pm}=0
$$

corresponding to the repulsive interaction between atoms in the BEC physical context (for definiteness we shall use throughout this interpretation of the Manakov system). At first we shall present the solution in the form obtained by the direct integration of this system.

\subsection{Direct method}

The direct method is based on the introduction of such new dependent variables that the functions $\psi_{ \pm}$are represented by the formulae [24]

$$
\left(\begin{array}{c}
\psi_{+} \\
\psi_{-}
\end{array}\right)=\sqrt{\rho} e^{i \Phi / 2}\left(\begin{array}{c}
\cos \frac{\theta}{2} e^{-i \phi / 2} \\
\sin \frac{\theta}{2} e^{i \phi / 2}
\end{array}\right) .
$$


Here $\rho(x, t)=\rho_{+}+\rho_{-}=\left|\psi_{+}\right|^{2}+\left|\psi_{-}\right|^{2}$ denotes the total density of the condensate and the angle $\theta(x, t)$ is the variable describing the relative density of two components $\left(\cos \theta=\left(\left|\psi_{+}\right|^{2}-\left|\psi_{-}\right|^{2}\right) / \rho\right) ; \Phi(x, t)$ has the meaning of the velocity potential of the in-phase motion and $\phi(x, t)$ is the potential of the relative (counter-phase) motion of the components. Accordingly, their gradients characterize the in-phase and relative velocities

$$
U(x, t)=\Phi_{x}, \quad v(x, t)=\phi_{x} .
$$

The variables $\rho(x, t), U(x, t)$ describe the in-phase motion of the components (density waves) and the variables $\theta(x, t), v(x, t)$ describe their counter-phase motion (polarization waves). Substitution of (2) and (3) into (11) yields the equations for the variables $\rho, U$, $\theta, v$, (see [25]) which can be written for future convenience in the form of conservation laws,

$$
\begin{aligned}
& \rho_{t}+\frac{1}{2}[\rho(U-v \cos \theta)]_{x}=0 \\
& U_{t}+\left[\frac{\rho_{x}^{2}}{4 \rho^{2}}-\frac{\rho_{x x}}{2 \rho}-\frac{\cot \theta}{2 \rho}\left(\rho \theta_{x}\right)_{x}+\frac{1}{4}\left(\theta_{x}^{2}+U^{2}+v^{2}\right)+2 \rho\right]_{x}=0 \\
& (\rho \cos \theta)_{t}+\frac{1}{2}[\rho(U \cos \theta-v)]_{x}=0 \\
& v_{t}+\left[\frac{1}{2} U v-\frac{1}{2 \rho \sin \theta}\left(\rho \theta_{x}\right)_{x}\right]_{x}=0 .
\end{aligned}
$$

The single-phase solution corresponds to the ansatz that all variables depend on $\xi=x-V t$ and the solution can be obtained in an explicit form under supposition that an integration constant appearing after integration of Eq. (7) equals to zero what means that the chemical potentials of both BEC components are equal to each other, that is

$$
\Phi=-2 \chi t+\Phi_{0}(\xi), \quad \phi=\phi(\xi) .
$$

where $\chi$ is the common value of the chemical potentials of both components. Then, as was shown in [16], the density $\rho(\xi)$ satisfies the equation

$$
\rho_{\xi}^{2}=4 \mathcal{R}(\rho)
$$

where

$$
\mathcal{R}(\rho)=\rho^{3}-\left(2 \chi+V^{2}\right) \rho^{2}+D \rho-\left(A^{2}+C^{2}\right)=\prod_{i=1}^{3}\left(\rho-\rho_{i}\right),
$$

we assume that $\rho_{1} \leq \rho_{2} \leq \rho_{3}$ and parameters $\chi, A, C$ and $D$ are the integration constants. The solution of (10) can be expressed in standard notation in terms of the Jacobi elliptic function,

$$
\rho(\xi)=\rho_{1}+\left(\rho_{2}-\rho_{1}\right) \operatorname{sn}^{2}\left(\sqrt{\rho_{3}-\rho_{1}}\left(\xi+\xi_{0}\right), m\right),
$$

where $m=\left(\rho_{2}-\rho_{1}\right) /\left(\rho_{3}-\rho_{1}\right)$. Thus, $\rho$ oscillates in the interval $0 \leq \rho_{1} \leq \rho \leq \rho_{2}$.

When the density $\rho(\xi)$ is known, the angle $\theta(\xi)$ can be found by integration of the equation

$$
\pm \frac{\sin \theta d \theta}{\sqrt{\left(\cos \theta-\cos \theta_{1}\right)\left(\cos \theta_{2}-\cos \theta\right)}}=2 R \frac{d \xi}{\rho(\xi)}
$$


where the parameters $R, \theta_{1}, \theta_{2}$ are related with previously introduced parameters by the relations

$$
\begin{aligned}
& R^{2} \equiv \rho_{1} \rho_{2} \rho_{3}=A^{2}+C^{2}, \quad \theta_{1}=\beta+\gamma, \quad \theta_{2}=\beta-\gamma, \\
& A=R \cos \gamma, \quad C=R \sin \gamma, \quad B=R \cos \beta,
\end{aligned}
$$

so that $\cos \theta$ oscillates in the interval $\cos \theta_{1} \leq \cos \theta \leq \cos \theta_{2}$. Equation (12) can be easily integrated to give

$$
\cos \theta(\xi)=\cos \theta_{1} \sin ^{2} \frac{X(\xi)}{2}+\cos \theta_{2} \cos ^{2} \frac{X(\xi)}{2},
$$

where

$$
X(\xi)=2 R \int_{\xi_{0}}^{\xi} \frac{d \xi^{\prime}}{\rho_{1}+\left(\rho_{2}-\rho_{1}\right) \operatorname{sn}^{2}\left(\sqrt{\rho_{3}-\rho_{1}} \xi^{\prime}, m\right)}+X_{0},
$$

$X_{0}$ is an integration constant.

At last, $U$ and $v$ are given by the formulae

$$
\begin{aligned}
& U(\xi)=2 V+\frac{2 R}{\rho(\xi)} \frac{\cos \beta \cos \theta(\xi)-\cos \gamma}{\sin ^{2} \theta(\xi)} \\
& v(\xi)=\frac{2 R}{\rho(\xi)} \frac{\cos \beta-\cos \gamma \cos \theta(\xi)}{\sin ^{2} \theta(\xi)}
\end{aligned}
$$

and their integration yields the phases $\Phi=-2 \chi t+\int U d x$ and $\phi=\int v d x$.

Two particular solution are of special interest. If $\gamma=0$, then $\theta=\beta=$ const and $v=0$, that is there is no relative motion of the components. In particular, if $\rho_{2}=\rho_{3}=\rho_{0}$, then the solution reduces to the standard Manakov dark soliton wave with

$$
\begin{aligned}
& \rho(x, t)=\rho_{0}\left\{1-\frac{1-\rho_{1} / \rho_{0}}{\cosh ^{2}\left[\sqrt{\rho_{0}-\rho_{1}}(x-V t)\right]}\right\}, \\
& U(x, t)=2\left(V-\rho_{0} \sqrt{\rho_{1}} / \rho(x, t)\right) .
\end{aligned}
$$

If soliton propagates through a quiescent condensate, that is $U \rightarrow 0, \rho \rightarrow \rho_{0}$ as $|x| \rightarrow \infty$, then $V=\sqrt{\rho_{1}}$ and we reproduce a well-known one-soliton solution. It does not depend on the parameter $\beta$, because in this case there is no dynamical difference between the components and the motion reduces effectively to a one-component dynamics: both components move in phase keeping the constant ratio of the densities.

The second important particular solution corresponds to the constant total density $\rho \equiv \rho_{0}$ when $\rho_{1}=\rho_{2}=\rho_{0}$, and hence $R=\rho_{0} \sqrt{\rho_{3}}$. In such a wave the components exchange their places in the counter-phase relative motion called a pure polarization dynamics. It is described by (15) with

$$
X(\xi)=2 \sqrt{\rho_{3}}\left(\xi-\xi_{0}\right)+X_{0} .
$$

The total flux of atoms $v_{+} \rho_{+}+v_{-} \rho_{-}=\rho_{0}(U-v \cos \theta) / 2=\rho_{0}\left(V-\sqrt{\rho_{3}} \cos \gamma\right)$ vanishes when $V=\sqrt{\rho_{3}} \cos \gamma$. According to Eq. (20), the wave number of the polarization wave equals to $k=2 \sqrt{\rho_{3}}$, hence $V$ is the phase velocity $V=\omega / k$ corresponding to the dispersion relation of the polarization waves given by

$$
\omega(k)=\frac{1}{2} k^{2} \cos \gamma, \quad V=\frac{\omega}{k}=\frac{1}{2} k \cos \gamma .
$$


On periodic solutions for the Manakov system

More general solutions which combine oscillations of the total density $\rho$ and of the polarization variable $\theta$ were considered in [16].

\subsection{Finite-gap method}

In this section we shall reproduce the main results of Wright's paper [15] with the goal to relate them with the results of the preceding section. For consistency, we shall slightly change the notation of [15] and make some additions to Wright's results.

The finite-gap method is based on the possibility to represent the Manakov system (11) as a compatibility condition of two linear systems (see [10])

$$
\Psi_{x}=\mathbb{U} \Psi, \quad \Psi_{t}=\mathbb{V} \Psi,
$$

that is from the condition

$$
\mathbb{U}_{t}-\mathbb{V}_{x}+[\mathbb{U}, \mathbb{V}]=0
$$

where $[\cdot, \cdot]$ is a commutator of matrices and

$$
\begin{aligned}
& \mathbb{U}=\left(\begin{array}{ccc}
F_{1}+F_{2} & G_{1} & G_{2} \\
H_{1} & -F_{1} & G_{3} \\
H_{2} & H_{3} & -F_{2}
\end{array}\right), \quad \mathbb{V}=\left(\begin{array}{ccc}
A_{1}+A_{2} & B_{1} & B_{2} \\
C_{1} & -A_{1} & B_{3} \\
C_{2} & C_{3} & -A_{2}
\end{array}\right),(22) \\
& F_{1}=F_{2}=-i \lambda, \quad G_{1}=i \psi_{+}, \quad G_{2}=i \psi_{-}, \quad G_{3}=0, \\
& H_{1}=-i \psi_{+}^{*}, \quad H_{2}=-i \psi_{-}^{*}, \quad H_{3}=0 ; \\
& A_{1}=-\frac{3}{2} i \lambda^{2}-\frac{1}{2} i\left|\psi_{+}\right|^{2}, \quad A_{2}=-\frac{3}{2} i \lambda^{2}-\frac{1}{2} i\left|\psi_{-}\right|^{2}, \quad B_{2}=\frac{3}{2} i \lambda \psi_{-}-\frac{1}{2} \psi_{-, x}, \quad B_{3}=\frac{1}{2} i \psi_{+}^{*} \psi_{-}, \\
& B_{1}=\frac{3}{2} i \lambda \psi_{+}-\frac{1}{2} \psi_{+, x}, \quad C_{2}=-\frac{3}{2} i \lambda \psi_{-}^{*}-\frac{1}{2} \psi_{-, x}^{*}, \quad C_{3}=\frac{1}{2} i \psi_{+} \psi_{-}^{*} . \\
& C_{1}=-\frac{3}{2} i \lambda \psi_{+}^{*}-\frac{1}{2} \psi_{+, x}^{*}, \quad
\end{aligned}
$$

Equations (22) can be cast to the matrix form

$$
\mathbb{W}_{x}=[\mathbb{U}, \mathbb{W}], \quad \mathbb{W}_{t}=[\mathbb{V}, \mathbb{W}],
$$

where $\mathbb{W}$ can be built from the basis solutions $\Psi_{0}$ of the systems (22) (see, e.g., [14]). The explicit formulae expressing the matrix elements of $\mathbb{W}$ in terms of $\Psi_{0}$ are presented in Appendix A. Let us define a characteristic polynomial of the matrix $\mathbb{W}$ by the equation

$$
\mathcal{Q}(\lambda, w)=i \operatorname{det}[i w \mathbb{I}-\mathbb{W}]=w^{3}+a(\lambda) w+b(\lambda),
$$

where $\mathbb{I}$ is the identity matrix. Then from the fact that $\mathbb{W}$ satisfies the equations (27) one can obtain the theorem that the coefficients of the polynomial $\mathcal{Q}$ do not depend on $x$ and $t$. Since these coefficients are expressed in terms of the matrix elements of $\mathbb{U}$ and $\mathbb{V}$, that is in terms of $\psi_{ \pm}$and their $x$-derivatives, we arrive at the conservation laws of the Manakov system (1). Simple proof of this theorem is presented for completeness in Appendix B. 
On periodic solutions for the Manakov system

Under supposition that the matrix elements of $\mathbb{W}$ are the second-degree polynomials in $\lambda$, Wright obtains the solution

$$
\mathbb{W}=\left(\begin{array}{ccc}
f_{1}+f_{2} & g_{1} & g_{2} \\
h_{1} & -f_{1} & g_{3} \\
h_{2} & h_{3} & -f_{2}
\end{array}\right)
$$

where

$$
\begin{aligned}
& f_{1}=-i \lambda^{2}-\frac{2}{3} i V \lambda-\frac{1}{3} i\left|\psi_{+}\right|^{2}+\frac{2}{9} i \chi, \\
& f_{2}=-i \lambda^{2}-\frac{2}{3} i V \lambda-\frac{1}{3} i\left|\psi_{-}\right|^{2}+\frac{2}{9} i \chi, \\
& g_{1}=i \psi_{+}\left(\lambda-\mu_{1}\right), \quad g_{2}=i \psi_{-}\left(\lambda-\mu_{2}\right), \quad g_{3}=\frac{1}{3} i \psi_{+}^{*} \psi_{-}, \\
& h_{1}=-i \psi_{+}^{*}\left(\lambda-\mu_{1}^{*}\right), \quad h_{2}=-i \psi_{-}^{*}\left(\lambda-\mu_{2}^{*}\right), \quad h_{3}=-\frac{1}{3} i \psi_{+} \psi_{-}^{*},
\end{aligned}
$$

$V$ and $\chi$ are constant parameters, and the coefficients of these polynomials satisfy the equations

$$
\begin{aligned}
& \psi_{+, x}=3 i \mu_{1} \psi_{+}+2 i V \psi_{+}, \quad \psi_{-, x}=3 i \mu_{2} \psi_{+}+2 i V \psi_{-}, \\
& \psi_{+, t}=-V \psi_{+, x}-i \chi \psi_{+}, \quad \psi_{-, t}=-V \psi_{-, x}-i \chi \psi_{-}, \\
& \mu_{1, x}=-3 i \mu_{1}^{2}-2 i V \mu_{1}-\frac{2}{3} i\left(\left|\psi_{+}\right|^{2}+\left|\psi_{-}\right|^{2}\right)+\frac{2}{3} i \chi \\
& \mu_{2, x}=-3 i \mu_{2}^{2}-2 i V \mu_{2}-\frac{2}{3} i\left(\left|\psi_{+}\right|^{2}+\left|\psi_{-}\right|^{2}\right)+\frac{2}{3} i \chi \\
& \mu_{1, t}=-V \mu_{1, x}, \quad \mu_{2, t}=-V \mu_{2, x} .
\end{aligned}
$$

From these equations one can obtain

$$
\left(\left|\psi_{+}\right|^{2}\right)_{x}=3 i\left|\psi_{+}\right|^{2}\left(\mu_{1}-\mu_{1}^{*}\right), \quad\left(\left|\psi_{-}\right|^{2}\right)_{x}=3 i\left|\psi_{-}\right|^{2}\left(\mu_{2}-\mu_{2}^{*}\right) .
$$

One can see that the variables $\mu_{1}, \mu_{2}$ as well as $\rho_{ \pm}=\left|\psi_{ \pm}\right|^{2}$ depend on $\xi=v-V t$ only, that is $V$ denotes the phase velocity of the wave and $\chi$ plays the role of the chemical potential in the BEC context. In this particular case of the second degree polynomials (30) in $\lambda$ the coefficients of the polynomial $\mathcal{Q}$ take the form

$$
\begin{aligned}
a(\widetilde{\lambda})= & -3\left[\widetilde{\lambda}^{2}-\frac{1}{9}\left(V^{2}+2 \chi\right)\right]^{2}-\left(\widetilde{\lambda}-\frac{1}{3} V\right) S-I_{3}, \\
b(\widetilde{\lambda})=2 & {\left[\widetilde{\lambda}^{2}-\frac{1}{9}\left(V^{2}+2 \chi\right)\right]^{3} } \\
& +\left[\left(\widetilde{\lambda}-\frac{1}{3} V\right) S+I_{3}\right]\left[\widetilde{\lambda}^{2}-\frac{1}{9}\left(V^{2}+2 \chi\right)\right]-\frac{1}{3} I_{4},
\end{aligned}
$$

where $\widetilde{\lambda}=\lambda-V / 3, S=I_{1}+I_{2}$, and $I_{1}, I_{2}, I_{3}, I_{4}$ are the integrals introduced by Wright:

$$
\begin{aligned}
& I_{1}=\left|\psi_{+}\right|^{2}\left(\mu_{1}^{*}+\mu_{1}+2 V / 3\right), \quad I_{2}=\left|\psi_{-}\right|^{2}\left(\mu_{2}^{*}+\mu_{2}+2 V / 3\right), \\
& I_{3}=\frac{1}{9}\left(\left|\psi_{+}\right|^{2}+\left|\psi_{-}\right|^{2}\right)^{2}-\left|\psi_{+}\right|^{2}\left|\mu_{1}\right|^{2}-\left|\psi_{-}\right|^{2}\left|\mu_{2}\right|^{2}-\frac{2}{9} \chi\left(\left|\psi_{+}\right|^{2}+\left|\psi_{-}\right|^{2}\right), \\
& I_{4}=\left|\psi_{+}\right|^{2}\left|\psi_{-}\right|^{2}\left|\mu_{1}-\mu_{2}\right|^{2} .
\end{aligned}
$$


It is important to notice that the polynomial $\mathcal{Q}$ depends on the sum $S$ of integrals $I_{1}$ and $I_{2}$ only rather than on them separately.

If we introduce the parameter $\widetilde{\beta}=\left(V^{2}+2 \chi\right) / 9$ and make a substitution $w=$ $\widetilde{\lambda}^{2}-\widetilde{\beta}+z$ in $\mathcal{Q}=w^{3}+a(\lambda) w+b(\lambda)$, then we obtain $\mathcal{Q}$ in new variables

$$
\mathcal{Q}=z^{3}+3\left(\widetilde{\lambda}^{2}-\widetilde{\beta}\right) z^{2}-\left[(\widetilde{\lambda}-V / 3) S+I_{3}\right] z-\frac{1}{3} I_{3}
$$

One can notice that this is a quadratic in $\widetilde{\lambda} z$ polynomial so that we arrive at the identity

$$
\mathcal{Q}=w^{3}+a(\lambda) w+b(\lambda) \equiv \frac{1}{27}\left[y^{2}+\mathcal{R}(\nu)\right]
$$

where $w, \lambda$ are related with the variables $y=3 z, \nu$ by the equations

$$
\lambda=\frac{1}{\nu}\left(\frac{y}{3}+\frac{S}{2}\right)-\frac{V}{3}, \quad w=\frac{1}{\nu^{2}}\left(\frac{y}{3}+\frac{S}{2}\right)^{2}-\frac{1}{9}\left(V^{2}+2 \chi\right)+\frac{\nu}{3},
$$

and

$$
\mathcal{R}(\nu)=\nu^{3}-\left(2 \chi+V^{2}\right) \nu^{2}+9\left(\frac{1}{3} V S-I_{3}\right) \nu-\frac{9}{4}\left(S^{2}+4 I_{4}\right) .
$$

Using the integrals (34), Wright has found useful expressions for the parameters $\mu_{1}, \mu_{2}$ which we write in the form

$$
\begin{aligned}
& \mu_{1}=\frac{1}{2}\left(\frac{I_{1}}{\left|\psi_{+}\right|^{2}}-\frac{2 V}{3}\right) \pm \frac{i \sqrt{\mathcal{S}}}{2 \rho\left|\psi_{+}\right|^{2}}+\frac{i \sqrt{\mathcal{R}(\rho)}}{3 \rho}, \\
& \mu_{2}=\frac{1}{2}\left(\frac{I_{2}}{\left|\psi_{-}\right|^{2}}-\frac{2 V}{3}\right) \mp \frac{i \sqrt{\mathcal{S}}}{2 \rho\left|\psi_{-}\right|^{2}}+\frac{i \sqrt{\mathcal{R}(\rho)}}{3 \rho},
\end{aligned}
$$

where the function $\mathcal{R}$ is the same as in (37), $\rho=\left|\psi_{+}\right|^{2}+\left|\psi_{-}\right|^{2}$ is the total density, and $\mathcal{S}$ is defined by the expression

$$
\mathcal{S}=4\left|\psi_{+}\right|^{2}\left|\psi_{-}\right|^{2} I_{4}-\left(I_{1}\left|\psi_{-}\right|^{2}-I_{2}\left|\psi_{+}\right|^{2}\right)^{2}
$$

Substitution of (38) in the sum of (32) yields the equation for $\rho$ :

$$
\rho_{x}^{2}=4 \mathcal{R}(\rho) .
$$

If we introduce the variable $\theta$ characterizing the relative density of the components according to

$$
1+\cos \theta=2 \cos ^{2} \frac{\theta}{2}=\frac{2\left|\psi_{+}\right|^{2}}{\rho}
$$

then we can find equation for $\theta$ with the use of (32) and (40)

$$
\begin{aligned}
& \left(\frac{d \cos \theta}{d x}\right)^{2}=\frac{36}{\rho^{4}} \mathcal{S} \\
& =-\frac{9}{\rho^{2}}\left[\left(S^{2}+4 I_{4}\right) \cos ^{2} \theta-2\left(I_{1}^{2}-I_{2}^{2}\right) \cos \theta+\left(I_{1}-I_{2}\right)^{2}-4 I_{4}\right] .
\end{aligned}
$$

Since $\rho$ and $\theta$ depend on $\xi=x-V t$ only, their $x$-derivatives coincide with $\xi$-derivatives. Hence, the functions $\mathcal{R}$ and $\mathcal{S}$ govern the evolution of $\rho$ and $\theta$ in the single-phase solution of the Manakov system. 
On periodic solutions for the Manakov system

Equations (40) and (42) can be identified with equations (9) and (12) of the preceding Section provided

$$
\begin{aligned}
& R^{2}=\frac{9}{4}\left(S^{2}+I_{4}\right), \quad A=\frac{3}{2} S=R \cos \gamma, \quad C=3 \sqrt{I_{4}}=R \sin \gamma, \\
& \cos \beta=\frac{I_{1}-I_{2}}{\sqrt{S^{2}+4 I_{4}}}, \quad \cos \gamma=\frac{S}{\sqrt{S^{2}+4 I_{4}}} .
\end{aligned}
$$

From the last two equations with account of $S=I_{1}+I_{2}$ we find

$$
I_{1}=\frac{R}{3}(\cos \gamma+\cos \beta), \quad I_{2}=\frac{R}{3}(\cos \gamma-\cos \beta)
$$

and

$$
\mathcal{S}=\frac{R^{2} \rho^{2}}{9}\left(\cos \theta-\cos \theta_{1}\right)\left(\cos \theta_{2}-\cos \theta\right)
$$

where $\theta_{1}=\beta+\gamma, \theta_{2}=\beta-\gamma($ see $(\underline{13}))$. Then velocities $v_{ \pm}=(U \mp v) / 2$ of two components can be written as

$$
v_{+}=V-\frac{3 I_{1}}{2 \rho \cos ^{2}(\theta / 3)}, \quad v_{-}=V-\frac{3 I_{2}}{2 \rho \sin ^{2}(\theta / 3)} .
$$

With the use of new parameters the equations (38) can be written as

$$
\begin{aligned}
& \mu_{1}=-\frac{v_{+}}{3} \pm \frac{i \sqrt{\mathcal{S}}}{2 \rho^{2} \cos ^{2}(\theta / 2)}+\frac{i \sqrt{\mathcal{R}}}{3 \rho}, \\
& \mu_{2}=-\frac{v_{-}}{3} \mp \frac{i \sqrt{\mathcal{S}}}{2 \rho^{2} \sin ^{2}(\theta / 2)}+\frac{i \sqrt{\mathcal{R}}}{3 \rho} .
\end{aligned}
$$

Here $\mathcal{R}=\mathcal{R}(\rho), \mathcal{S}=\mathcal{S}(\rho, \cos \theta)$ and both $\rho$ and $\cos \theta$ are functions of $\xi=x-V t$ given by (11) and (15), correspondingly. Hence, equations (47) define paths of the auxiliary spectrum points $\mu_{1,2}$ in the complex plane $\lambda$ corresponding to the single-phase evolution of $\psi_{ \pm}$according to the Manakov system.

The single-phase solution is parameterized here by the integrals $I_{1}, I_{2}, I_{3}, I_{4}$ and by the velocity $V$ and the chemical potential $\chi$. However, in the finite-gap integration method a special role is played by the parameters defining the spectral curve. In our case such a curve is given by the equation

$$
\mathcal{Q}(\lambda, w)=w^{3}+a(\lambda) w+b(\lambda)=0
$$

which defines the dependence of the complex variable $w$ on the complex variable $\lambda$. As we know, this equations has constant coefficients expressed in terms of $S=I_{1}+I_{2}$, $I_{3}, I_{4}, V$ and $\chi$ (see (33) $)$. Thus, there are only five independent parameters which cannot parameterize completely the single-phase solution. Another set of convenient parameters is presented by the branching points of the curve $w=w(\lambda)$ defined by (48). These points are determined by the condition that the discriminant of (48) vanishes. The discriminant can be defined as

$$
\Delta(\lambda)=-\frac{1}{9}\left[4 a^{3}(\lambda)+27 b^{2}(\lambda)\right]
$$


and it equals to

$$
\begin{aligned}
& \Delta(\lambda)=\left(S \lambda+I_{3}\right)^{2}\left[\left(\lambda^{2}+\frac{2}{3} V \lambda-\frac{2}{9} \chi\right)^{2}+\frac{4}{9}\left(S \lambda+I_{3}\right)\right] \\
& +2\left(\lambda^{2}+\frac{2}{3} V \lambda-\frac{2}{9} \chi\right)\left[2\left(\lambda^{2}+\frac{2}{3} V \lambda-\frac{2}{9} \chi\right)^{2}+\left(S \lambda+I_{3}\right)\right] I_{4}-\frac{1}{3} I_{4}^{2} .
\end{aligned}
$$

This is sixth-degree polynomial in $\lambda$ and equation $\Delta(\lambda)=0$ has six $\operatorname{roots} \lambda_{i}, i=1, \ldots, 6$, representing the branching points of the function $w(\lambda)$ in the complex $\lambda$-plane. They are not independent of each other since they depend on five free parameters only. On the other hand, our initial parameters $S=I_{1}+I_{2}, I_{3}, I_{4}, V$ and $\chi$ can be, in principle, expressed in terms of $\lambda_{i}, i=1, \ldots, 6$, and, hence, these branching points provide the most natural parametrization of the solution in the context of the finite-gap integration method. In particular, using the first two terms of the expansion

$$
\Delta(\lambda)=\left(4 I_{4}+S^{2}\right) \lambda^{6}+\frac{2}{3}\left[3 I_{3} S+2\left(6 I_{4}+S^{2}\right) V\right] \lambda^{5}+\ldots
$$

we find the relation

$$
V=-\frac{3\left(4 I_{4}+S^{2}\right)}{4\left(6 I_{4}+S^{2}\right)} \sum_{i=1}^{6} \lambda_{i}-\frac{3 I_{3} S}{2\left(6 I_{4}+S^{2}\right)} .
$$

As one can see from (50), the situation simplifies drastically for $I_{4}=0$. Let us consider this particular case in some detail. The discriminant reduces to

$$
\Delta(\lambda)=\left(S \lambda+I_{3}\right)^{2} P_{0}(\lambda),
$$

where

$$
\begin{aligned}
P_{0}(\lambda) & =\left(\lambda^{2}+\frac{2}{3} V \lambda-\frac{2}{9} \chi\right)^{2}+\frac{4}{9}\left(S \lambda+I_{3}\right) \\
& =\lambda^{4}+\frac{4}{3} V \lambda^{3}+\frac{4}{9}\left(V^{2}-\chi\right) \lambda^{2}+\frac{4}{27}(3 S-2 V \chi) \lambda+\frac{4}{9} I_{3}+\frac{4}{81} \chi^{2}
\end{aligned}
$$

is the forth-degree polynomial in $\lambda$ with zeroes $\lambda_{0 i}, i=1, \ldots, 4$. The doubled zero of the discriminant equals to $\lambda_{05}=\lambda_{06}=-I_{3} / S$. Then (151) reduces to

$$
V=-\frac{3}{4} \sum_{i=0}^{4} \lambda_{0 i}
$$

and that is confirmed by the Viète formulae for (53). Calculation of the Ferrari resolvent (see [27] and [6]) for the polynomial $P_{0}(\lambda)$ yields

$$
\begin{aligned}
\mathcal{R}_{0}(\widetilde{\nu})= & \left(\frac{4}{9}\right)^{3}\left\{\left(\frac{9}{4} \widetilde{\nu}\right)^{3}-\left(V^{2}+2 \chi\right)\left(\frac{9}{4} \widetilde{\nu}\right)^{2}\right. \\
& \left.+9\left(\frac{1}{3} S V-I_{3}\right)\left(\frac{9}{4} \widetilde{\nu}\right)-\frac{9}{4} S^{2}\right\} .
\end{aligned}
$$


This coincides (up to a constant numerical factor) with the polynomial (37) where it is assumed that $I_{4}=0$ and $\nu=(9 / 4) \widetilde{\nu}$. Consequently, we immediately obtain the relations between the zeroes $\rho_{0 i}$ of (37) (with $I_{4}=0$ ) and the zeroes $\lambda_{0 i}$ of the polynomial $P_{0}(\lambda)$,

$$
\begin{aligned}
& \rho_{01}=\left(\lambda_{01}-\lambda_{02}-\lambda_{03}+\lambda_{04}\right)^{2} / 9, \\
& \rho_{02}=\left(\lambda_{01}-\lambda_{02}+\lambda_{03}-\lambda_{04}\right)^{2} / 9, \\
& \rho_{03}=\left(\lambda_{01}+\lambda_{02}-\lambda_{03}-\lambda_{04}\right)^{2} / 9
\end{aligned}
$$

(see [6, 8, 15]). Here the zeroes are ordered in such a way that for $\lambda_{01} \leq \lambda_{02} \leq \lambda_{03} \leq \lambda_{04}$ we get $\rho_{01} \leq \rho_{02} \leq \rho_{03}$. These formulae suggest that the case with $I_{4}=0$ reduces to the situation known for the theory of periodic solutions of the one-component NLS equation [6, 8] with the polynomial $P_{0}(\lambda)$ playing the role of the polynomial appearing in the elliptic spectral curve in the NLS finite-gap theory. This suppositions is supported by noticing that in the case with $I_{4}=0$ the angle $\gamma$ defined by Eq. (43) vanishes that is in these periodic solutions there is no variations of the relative densities of the components and dynamical difference between them disappears. Formally we have $\theta=\theta_{1}=\theta_{2}=\beta=$ const and consequently $\mathcal{S} \equiv 0$. Then from (39) we get

$$
\frac{I_{1}}{\left|\psi_{+}\right|^{2}}=\frac{I_{2}}{\left|\psi_{-}\right|^{2}}=\frac{I_{1}+I_{2}}{\left|\psi_{+}\right|^{2}+\left|\psi_{-}\right|^{2}}=\frac{S}{\rho},
$$

hence

$$
\mu_{1}=\mu_{2}=\mu=\frac{S}{2 \rho}-\frac{V}{3}+\frac{i \sqrt{\mathcal{R}(\rho)}}{3 \rho} .
$$

Now the polynomial $P_{0}(\lambda)$ satisfies the identity (see [6, 8])

$$
P_{0}(\lambda)=f_{0}^{2}(\lambda)-\frac{4}{9} \rho(\lambda-\mu)\left(\lambda-\mu^{*}\right)
$$

where

$$
f_{0}(\lambda)=\lambda^{2}+\frac{2}{3} V \lambda+\frac{2}{9}(\rho-\chi)
$$

what equals to the value of $i f_{1,2}$ defined by (30) for the case $I_{4}=0$. Consequently, equations for $\mu_{1, x}$ and $\mu_{2, x}$ in (31) reduce to

$$
\mu_{x}=-i\left(3 \mu^{2}+2 V \mu+\frac{2}{3}(\rho-\chi)\right)=-3 i f_{0}(\mu)=-3 i \sqrt{P_{0}(\mu)},
$$

that is to the well-known Dubrovin equation for a single-phase solution of the NLS equation (see, e.g., [6, 7, 8]).

Thus, we have reproduced the main equations of the preceding Section obtained by a direct method in [16] by means of the finite-gap integration method developed by Wright [15] and have related the finite-gap equations for the Manakov system with the finite-gap equations for the NLS equation in the limit of density waves corresponding to $I_{4}=0$. The advantage of the finite-gap integration method is that the branching points of the appearing in this method spectral curve provide the Riemann invariants of the Whitham modulation equations. In the next Section we shall consider Whitham equation for the particular cases of density and polarization waves. 
On periodic solutions for the Manakov system

\section{Modulation theory}

\subsection{Whitham equations for density waves}

At first let us consider the dispersionless limit when the dispersion effects are neglected that is all higher order $x$-derivatives are omitted in the system (44)-(7):

$$
\begin{aligned}
& \rho_{t}+\frac{1}{2}[\rho(U-v \cos \theta)]_{x}=0, \\
& U_{t}+\left(\frac{1}{4} U^{2}+\frac{1}{4} v^{2}+2 \rho\right)_{x}=0, \\
& (\rho \cos \theta)_{t}+\frac{1}{2}[\rho(U \cos \theta-v)]_{x}=0, \\
& v_{t}+\frac{1}{2}(U v)_{x}=0 .
\end{aligned}
$$

If we introduce variables $r_{W}=\rho, s_{W}=\rho \cos \theta, k_{W}=U / 2, d_{W}=-v / 2$, then these equations transform to the dispersionless limit of Manakov equations

$$
\left(\begin{array}{c}
k_{W} \\
d_{W} \\
r_{W} \\
s_{W}
\end{array}\right)_{t}+\left(\begin{array}{cccc}
k_{W} & d_{W} & 1 & 0 \\
d_{W} & k_{W} & 0 & 0 \\
r_{W} & s_{W} & k_{W} & d_{W} \\
s_{W} & r_{W} & d_{W} & k_{W}
\end{array}\right)\left(\begin{array}{c}
k_{W} \\
d_{W} \\
r_{W} \\
s_{W}
\end{array}\right)_{x}=0
$$

obtained by Wright [23].

We are interested here in modulations of the density wave along which the conditions $v=0$ and $\theta=\beta=$ const hold. Consequently, the first and the third equations of the system (61) coincide with each other in this case and the fourth equation is fulfilled identically. Thus, we remain with two equations for $\rho$ and $U$ only which coincide with the Euler equations for polytropic gas flow with the ratio of specific heats equal to 2 (or to "shallow water equations")

$$
\rho_{t}+(\rho \widetilde{U})_{x}=0, \quad \widetilde{U}_{t}+\widetilde{U} \widetilde{U}_{x}+\rho_{x}=0,
$$

where $\widetilde{U}=U / 2$. Equations for this system in the Riemann invariant form are well known and can be written as

$$
\lambda_{1, t}+\frac{1}{4}\left(3 \lambda_{1}+\lambda_{2}\right) \lambda_{1, x}=0, \quad \lambda_{2, t}+\frac{1}{4}\left(\lambda_{1}+3 \lambda_{2}\right) \lambda_{2, x}=0,
$$

where

$$
\lambda_{1}=-\frac{1}{3}(\widetilde{U}-2 \sqrt{\rho}), \quad \lambda_{2}=-\frac{1}{3}(\widetilde{U}+2 \sqrt{\rho})
$$

are the Riemann invariants.

We are interested in demonstration that these Riemann invariants can be obtained as the branching points of the spectral curve, that is, in the density wave limit, as the zeroes of the polynomial $P_{0}(\lambda)$ defined in (53). To this end, we express the coefficients of $P_{0}(\lambda)$ in terms of $\rho$ and $U$ assuming that the merging zeroes of $\mathcal{R}(\nu)$ are equal to $\rho_{1}=\rho_{2}=\rho$. From (10) and (17) we get

$$
2 \rho+\rho_{3}=2 \chi+V^{2}, \quad U=2\left(V-\sqrt{\rho_{3}}\right),
$$


consequently $\sqrt{\rho_{3}}=V-U / 2$ and

$$
\chi=\rho+\frac{1}{8} U^{2}-\frac{1}{2} U V .
$$

Then we have

$$
\frac{9}{4} S^{2}=\rho^{2} \rho_{3}=\rho^{2}(V-U / 2)^{2},
$$

and in taking the square root we have to choose such a sign that two of the resulting roots $\lambda_{i}$ do not depend on $V$ according to the requirement that in the dispersionless limit the phase velocity of the wave becomes irrelevant parameter. The final result will show that the appropriate choice of sign corresponds to

$$
S=\frac{2}{9} \rho(U / 2-V) .
$$

At last, $\mu=S /(2 \rho)-V / 3=(U / 2-2 V) / 3$ and definition of $I_{3}$ in (34) leads to

$$
I_{3}=\frac{1}{9} \rho^{2}-\mu^{2} \rho-\frac{2}{9} \mu \rho=-\frac{1}{18}\left(U^{2}-6 U V+8 V^{2}+2 \rho\right) .
$$

Substitution of these values of the parameters into (53) yields

$$
P_{0}(\lambda)=\frac{1}{1296}(6 \lambda-U+4 V)^{2}\left(36 \lambda^{2}+12 U \lambda+U^{2}-16 \rho\right)
$$

and the roots of this polynomial are equal to (to simplify the notation, in what follows we omit zero in the index of $\lambda_{0 i}$ for the case $I_{4}=0$ )

$$
\begin{aligned}
& \lambda_{1}=\frac{1}{6}(-U-4 \sqrt{\rho}), \quad \lambda_{2}=\frac{1}{6}(-U+4 \sqrt{\rho}), \\
& \lambda_{3}=\lambda_{4}=\frac{1}{6}(U-4 V) .
\end{aligned}
$$

As we see, two first roots coincide with the dispersionless Riemann invariants (65). The last two roots correspond to the boundary of the Whitham oscillatory region with the smooth non-oscillatory region and they permit one to evaluate the velocity $V$ (and, hence, wavenumber) of the modulated wave with vanishing amplitude at this boundary (see [28]).

In a nonlinear modulated density wave the four zeroes $\lambda_{i}, i=1,2,3,4$, of the equation $P_{0}(\lambda)=0$ play the role of the Riemann invariants and the Whitham equations for them can be obtained by a simple modification of the method suggested in [21, 22]. From (23) and (27) one can find the identity

$$
\begin{aligned}
& \frac{\partial}{\partial t}\left(\frac{G_{1}}{g_{1}}\right)-\frac{\partial}{\partial x}\left(\frac{B_{1}}{g_{1}}\right) \\
& =\frac{1}{g_{1}^{2}}\left[\left(B_{2} H_{3}-G_{2} C_{3}\right) g_{1}+\left(G_{1} C_{3}-B_{1} H_{3}\right) g_{2}+\left(B_{1} G_{2}-G_{1} B_{2}\right) h_{3}\right],
\end{aligned}
$$

and similar identities can be obtained for other ratios of the matrix elements. In case of single-phase periodic solution, the right-hand side of this identity vanishes as it follows from (30) and (31). Hence, averaging of (171) over space intervals much less than a 
characteristic length of change of the slowly varying parameters yields the generating function of the averaged conservation laws

$$
\frac{\partial}{\partial t}\left\langle\frac{G_{1}}{\widetilde{g}_{1}}\right\rangle-\frac{\partial}{\partial x}\left\langle\frac{B_{1}}{\widetilde{g}_{1}}\right\rangle=0
$$

where we have made the replacement

$$
g_{1} \mapsto \widetilde{g}_{1}=\frac{g_{1}}{\sqrt{P_{0}(\lambda)}}
$$

to take into account the condition that during slow evolution the identity

$$
P_{0}(\lambda)=-f_{1}^{2}(\lambda)-\frac{4}{9} g_{1}(\lambda) h_{1}(\lambda)
$$

must hold (see [8]). This identity can be easily checked for the density waves with $I_{4}=0$. Hence, the imposed condition (74) means that the polarization excitations are not excited during slow evolution of the nonlinear modulated wave. Averaging can be performed according to the rule

$$
\left\langle\frac{1}{\lambda_{i}-\mu(\xi)}\right\rangle=\frac{1}{L} \int_{0}^{L} \frac{d x}{\lambda_{i}-\mu(x)}=\frac{1}{L} \oint \frac{d \mu}{2\left(\lambda_{i}-\mu\right) \sqrt{P_{0}(\mu)}}=-\frac{2}{L} \frac{\partial L}{\partial \lambda_{i}}
$$

where

$$
L=\oint \frac{d \mu}{3 \sqrt{-P_{0}(\mu)}}=\frac{2 K(m)}{3 \sqrt{\left(\lambda_{3}-\lambda_{1}\right)\left(\lambda_{4}-\lambda_{2}\right)}},
$$

$K(m)$ is the complete elliptic integral of the first kind and

$$
m=\frac{\rho_{2}-\rho_{1}}{\rho_{3}-\rho_{1}}=\frac{\left(\lambda_{2}-\lambda_{1}\right)\left(\lambda_{4}-\lambda_{3}\right)}{\left(\lambda_{3}-\lambda_{1}\right)\left(\lambda_{4}-\lambda_{2}\right)} .
$$

Differentiation of the slowly varying functions $\lambda_{i}(x, t)$ in (172) and taking the limits $\lambda \rightarrow \lambda_{i}$ yields the Whitham equations

$$
\left\langle\frac{1}{\lambda_{i}-\mu}\right\rangle \frac{\partial \lambda_{i}}{\partial t}-\left(\frac{3}{2}-V\left\langle\frac{1}{\lambda_{i}-\mu}\right\rangle\right) \frac{\partial \lambda_{i}}{\partial x}=0, \quad i=1,2,3,4
$$

or

$$
\frac{\partial \lambda_{i}}{\partial t}+v_{i} \frac{\partial \lambda_{i}}{\partial x}=0, \quad i=1,2,3,4
$$

where

$$
v_{i}=V-\frac{3}{2}\left\langle\frac{1}{\lambda_{i}-\mu}\right\rangle^{-1}=V+\frac{3}{4} \frac{L}{\partial_{i} L}, \quad \partial_{i}=\frac{\partial}{\partial \lambda_{i}},
$$

are the Whitham velocities. Taking into account (154), we can present (179) in the general form

$$
v_{i}=\left(1-\frac{L}{\partial_{i} L} \partial_{i}\right) V, \quad i=1,2,3,4
$$

As is known, it can be obtained from the conservation law of the number of waves (see [17])

$$
k_{t}+(k V)_{x}=0,
$$


where $k=2 \pi / L$ is a local wave vector, provided both $k$ and the phase velocity $V$ are expressed in term of the Riemann invariants $\lambda_{i}$ as that occurs in the finite-gap integration method, and there is no any additional restriction imposed on them. Just such a situation is realized in our case of density waves with $I_{4}=0$ when the periodic solution is parameterized by four constants $\chi, V, S, I_{3}$ equivalent to the set of the Riemann invariants $\lambda_{i}, i=1,2,3,4$. It is worth noticing that the Whitham equations coincide in this case with those for a periodic solution of one-component NLS equation obtained in [19, 20] what is physically natural since when a relative motion of two components of the condensate is absent then they become dynamically indistinguishable and their motion can be described by evolution of the total density $\rho$ and the in-phase velocity $2 U$.

Now let us turn to the opposite limit of purely polarization wave when the total density is constant and only a relative motion of the components is effective.

\subsection{Whitham equations for polarization waves}

The polarization wave solution is described by the formulae (15) and (20) which can be written in the form

$$
\begin{aligned}
& \cos \theta=\cos \beta \cos \gamma+\sin \beta \sin \gamma \cos [k(x-V t)], \\
& U=2 V-\frac{k}{2}\left[\frac{\cos \gamma+\cos \beta}{1+\cos \theta}+\frac{\cos \gamma-\cos \beta}{1-\cos \theta}\right], \\
& v=\frac{k}{2}\left[\frac{\cos \gamma+\cos \beta}{1+\cos \theta}-\frac{\cos \gamma-\cos \beta}{1-\cos \theta}\right],
\end{aligned}
$$

where the condition that the total flux $j=\rho_{+} v_{+}+\rho_{-} v_{-}$vanishes yields the relation

$$
V=\frac{1}{2} k \cos \gamma
$$

due to which the equation (4) with $\rho=$ const is fulfilled identically. Substitution of (82) -(84) into (5) and (77) yields

$$
U_{t}+\left(V U-V^{2}+k^{2} / 4\right)_{x}=0, \quad v_{t}+(V v)_{x}=0 .
$$

For strictly periodic waves these equations reduce to $U_{t}+V U_{x}=0$ and $v_{t}+V v_{x}=0$ with $V=$ const in agreement with $U=U(x-V t)$ and $v=v(x-V t)$ in such a solution of the Manakov system. Averaging can be performed with the use of the easily proven formulae

$$
\begin{aligned}
& \left\langle\frac{1}{1 \pm \cos \theta}\right\rangle=\frac{1}{|\cos \gamma \pm \cos \beta|}, \\
& \left\langle\frac{1}{(1 \pm \cos \theta)^{2}}\right\rangle=\frac{1 \pm \cos \beta \cos \gamma}{|\cos \gamma \pm \cos \beta|^{3}} .
\end{aligned}
$$

As a result we get

$$
\langle U\rangle=\left\{\begin{array}{cc}
2 V, \quad \cos \beta>\cos \gamma, \\
2 V-k, \quad \cos \beta>\cos \gamma,
\end{array} \quad\langle v\rangle=\left\{\begin{array}{cc}
k, & \cos \beta>\cos \gamma \\
0, & \cos \beta>\cos \gamma
\end{array}\right.\right.
$$


and the averaged equations (86) reduce to

$$
k_{t}+(V k)_{x}=0, \quad V_{t}+V V_{x}+\frac{1}{4} k k_{x}=0,
$$

where the first equation is the Whitham equation for conservation of number of waves which is implied in the Whitham theory of modulations. These equations coincide with the compressible gas dynamics equations for the polytropic flow with the ratio of specific heats equal to 3. Well-known transformation to the Riemann invariant form yields

$$
r_{1, t}+r_{1} r_{1, x}=0, \quad r_{2, t}+r_{2} r_{2, x}=0,
$$

where Riemann invariants are defined by the formulae

$$
r_{1}=V+k / 2=\frac{1}{2} k(\cos \gamma+1), \quad r_{2}=V-k / 2=\frac{1}{2} k(\cos \gamma-1),
$$

so that the physical variables are expressed as

$$
V=\frac{k}{2} \cos \gamma=\frac{1}{2}\left(r_{1}+r_{2}\right), \quad k=r_{1}-r_{2} .
$$

It is easy to see that $r_{1}>0$ and $r_{2}<0$.

Equation (82) gives $\langle\cos \theta\rangle=\cos \beta \cos \gamma$ and averaging of (66) yields

$$
(\cos \beta \cos \gamma)_{t}-\frac{1}{2}\left(k \cos \beta \sin ^{2} \gamma\right)_{x}=0
$$

or, after simple manipulations with the use of (85) and (91),

$$
(\cos \beta)_{t}+\frac{2}{r_{1}+r_{2}}\left(r_{1} r_{2} \cos \beta\right)_{x}=0 .
$$

This equation can be easily transformed to the diagonal form by means of introduction of the third Riemann invariant according to

$$
r_{3}=\left(\frac{r_{1} r_{2}}{r_{1}-r_{2}}\right)^{2} \cos \beta=\frac{k^{2}}{16} \sin ^{4} \gamma \cos \beta
$$

As a result we obtain

$$
r_{3, t}+\frac{2 r_{1} r_{2}}{r_{1}+r_{2}} r_{3, x}=0
$$

Thus, we have transformed the modulation equations for a polarization wave to the Riemann diagonal form (90), (95). The initial value problem can be easily solved due to this reduction: if the initial data for $k(x, 0), V(x, 0)$, and $\beta(x, 0)$ are given, then the initial data for $r_{1}(x, 0), r_{2}(x, 0), r_{3}(x, 0)$ are also known. Then well-known solutions of two independent Hopf equations (90) yield $r_{1}(x, t)$ and $r_{2}(x, t)$ for $t>0$. At last, a linear with respect to $r_{3}$ equation (95) with known variable coefficients can be solved by standard methods and that yields the complete solution of the Cauchy problem. Applications of this method to concrete physical problems will be given elsewhere. 


\section{Conclusion}

We have shown that the periodic solution of the Manakov system obtained by a direct method in [16] actually coincides with the solution obtained by the finite-gap integration method in [15]. The method of Wright has the advantage that it provides the parametrization of the solution suitable for derivation of the Whitham modulation equations for the density waves with in-phase motion of the components. However, this parametrization in terms of the branching points of the spectral curve corresponding to the solution is not enough for description of the polarization wave. In Wright's method this manifests itself in the fact that the coefficients of the spectral curve depend of the sum $I_{1}+I_{2}$ of the integrals whereas the polarization wave is parameterized by the values of $I_{1}$ and $I_{2}$ separately. Consequently, the modulation equations for the polarization wave have been derived by the original Whitham method of averaging of the conservation laws without use of the complete integrability of the Manakov system. In spite of that, the resulting equations can be transformed to the Riemann diagonal form, as it would be naturally to expect for the completely integrable equations. The results obtained provide the basis for applications of the theory to the problems of nonlinear dynamics of Bose-Einstein condensates related with recent experiments [29, 30, 31].

\section{Appendix A}

The systems (22) have three basis solutions which we denote as columns $\Psi^{(i)}=\left(\Psi_{j}^{(i)}\right)^{T}$, $i, j=1,2,3$. They constitute the fundamental representation of the group $S U(3)$. The traceless matrix $\mathbb{W}=\left(W_{i j}\right)$ is expressed in terms of eight independent variables constituting the irreducible adjoint representation 8 of $S U(3)$ (see [14])

$$
\mathbb{W}=\left(\begin{array}{ccc}
\left(\mathcal{F}_{123}-\mathcal{F}_{132}\right) / 3 & -\mathcal{F}_{113} / 2 & \mathcal{F}_{112} / 2 \\
\mathcal{F}_{223} / 2 & -\left(2 \mathcal{F}_{123}+\mathcal{F}_{132}\right) / 3 & \mathcal{F}_{122} \\
\mathcal{F}_{233} & -\mathcal{F}_{133} & \left(\mathcal{F}_{123}+2 \mathcal{F}_{132}\right) / 3
\end{array}\right)
$$

where among 27 elements

$$
\mathcal{F}_{i j k}=\Psi_{i}^{(1)} \Psi_{j}^{(2)} \Psi_{k}^{(3)}+\Psi_{j}^{(1)} \Psi_{i}^{(2)} \Psi_{k}^{(3)}-\Psi_{k}^{(1)} \Psi_{j}^{(2)} \Psi_{i}^{(3)}-\Psi_{k}^{(1)} \Psi_{i}^{(2)} \Psi_{j}^{(3)}
$$

only 8 are independent and they are chosen as $\mathcal{F}_{112}, \mathcal{F}_{113}, \mathcal{F}_{122}, \mathcal{F}_{123}, \mathcal{F}_{132}, \mathcal{F}_{133}, \mathcal{F}_{223}, \mathcal{F}_{233}$. One can easily check that the matrix (A.1) satisfies (27), if $\Psi_{j}^{(i)}$ satisfy (22).

\section{Appendix B}

We shall follow here the exposition given in [26].

Let us present the fundamental solutions of (22) as a matrix

$$
\mathbb{F}(x, t, \lambda)=\left(\mathbb{F}_{i j}\right)=\left(\left(\Psi_{j}^{(i)}\right)\right.
$$

normalized by the initial data condition

$$
\mathbb{F}(0,0, \lambda)=\mathbb{I} .
$$


It is easy to check that if $\mathbb{W}(x, t, \lambda)$ is the solution of (27), then the product of two matrices

$$
\widetilde{\mathbb{F}}=\mathbb{W}(x, t, \lambda) \cdot \mathbb{F}(x, t, \lambda)
$$

is also the solution of (22). But any solution $\widetilde{\mathbb{F}}$ is a linear combination of the fundamental solutions

$$
\widetilde{\mathbb{F}}(x, t, \lambda)=\mathbb{F}(x, t, \lambda) \cdot \mathbb{G}(\lambda),
$$

where the matrix $\mathbb{G}$ can be determined from the normalization condition (B.2) by means of comparison of the expression (by (B.4)

$$
\widetilde{\mathbb{F}}(0,0, \lambda)=\mathbb{I} \cdot \mathbb{G}(\lambda)=\mathbb{G}(\lambda)
$$

with (by (B.3))

$$
\widetilde{\mathbb{F}}(0,0, \lambda)=\mathbb{W}(0,0, \lambda) \cdot \mathbb{F}(0,0, \lambda)=\mathbb{W}(0,0, \lambda) \cdot \mathbb{I}=\mathbb{W}(0,0, \lambda),
$$

that is $\mathbb{G}=\mathbb{W}(0,0, \lambda)$ and hence $\mathbb{F}(x, t, \lambda) \cdot \mathbb{W}(0,0, \lambda)=\mathbb{W}(x, t, \lambda) \cdot \mathbb{F}(x, t, \lambda)$ or

$$
\mathbb{W}(x, t, \lambda)=\mathbb{F}(x, t, \lambda) \cdot \mathbb{W}(0,0, \lambda) \cdot \mathbb{F}^{-1}(x, t, \lambda) .
$$

Consequently, we get

$$
i w \mathbb{I}-\mathbb{W}(x, t, \lambda)=\mathbb{F}(x, t, \lambda) \cdot[i w \mathbb{I}-\mathbb{W}(0,0, \lambda)] \cdot \mathbb{F}^{-1}(x, t, \lambda)
$$

and taking determinant of this identity we arrive at the statement of the theorem.

\section{References}

[1] Belokolos E D, Bobenko A I, Enol'skii V Z, Its A R and Matveev V B 1994 Algebro-Geometric Approach to Nonlinear Integrable Equations (Berlin: Springer)

[2] Zakharov V E and Shabat A B 1971 Zh. Exp. Teor. Fiz. 61118 [Zakharov V E and Shabat A B 1972 Sov. Phys. JETP 34 62]

[3] Its A R and Kotlyarov V P 1976 Dokl. Akad. Nauk UkrSSR A 11965

[4] Ma Y C and Ablowitz M J 1981 Stud. Appl. Math. 65113

[5] Tracy E R and Chen H H 1988 Phys. Rev. A 37815

[6] Kamchatnov A M 1990 J. Phys. A: Math. Gen. 232945

[7] Kamchatnov A M 1997 Phys. Rep. 286199

[8] Kamchatnov A M 2000 Nonlinear Periodic Waves and Their Modulations: An Introductory Course (Singapore: World Scientific)

[9] Ablowitz M J, Kaup D J, Newell A C and Segur H 1974 Stud. Appl. Math. 53249

[10] Manakov S V 1973 Zh. Eksp. Teor. Fiz. 65505 [Manakov S V 1974 Sov. Phys. JETP 38 248]

[11] Porubov A V and Parker D F 1999 Wave Motion 2997

[12] Christiansen P L, Eilbeck J C, Enolskii V Z and Kostov N A 2000 Proc. Roy. Soc. A 4562263

[13] Eilbeck J C, Enolskii V Z and Kostov N A 2000 J. Math. Phys. 418236

[14] Shin H J 2003 J. Phys. A: Math. Gen. 364113

[15] Wright O C 2013 Physica D 2641

[16] Kamchatnov A M 2013 EPL 10360003

[17] Whitham G B 1974 Linear and Nonlinear Waves (New York: Wiley)

[18] Flaschka H, Forest M G and McLaughlin D W 1980 Commun. Pure Appl. Math. 33739 
[19] Forest M G and Lee J E 1986 in Oscillation Theory, Computation and Methods of Compensated Compactness eds Dafermos C, Erickson J L, Kinderlehrer D and Slemrod M, IMA Volumes on Mathematics and its Applications 2 (New York: Springer)

[20] Pavlov M V 1987 Teor. Mat. Fiz. 71351 [Theor. Math. Phys. 71 584]

[21] Kamchatnov A M 1990 Zh. Eksp. Teor. Fiz. 97144 [Kamchatnov A M 1990 Sov. Phys. JETP 70 $80]$

[22] Kamchatnov A M 1994 Phys. Lett. A 186387

[23] Wright O C 1995 Physica D 821

[24] Kasamatsu K, Tsubota M and Ueda M 2005 Phys. Rev. A 71043611

[25] Kamchatnov A M, Kartashov Y V, Larré P-É and Pavloff N 2013 Nonlinear polarization waves in a two-component Bose-Einstein condensate Preprint cond-mat.quant-gas/1308.0784

[26] Dubrovin B A, Krichever I M and Novikov S P 1985 in Integrable Systems-I, Modern Problems of Mathematics, Fundamental Directions, 4179 (Moscow: VINITI) (in Russian)

[27] Van der Waerden B L 1971 Algebra (Berlin: Springer)

[28] Gurevich A V and Pitaevskii L P 1973 Zh. Eksp. Teor. Fiz. 65590 [Gurevich A V and Pitaevskii L P 1974 Sov. Phys. JETP 38 291]

[29] Hamner C, Chang J J, Engels P and Hoefer M A 2011 Phys. Rev. Lett. 106065302

[30] Hoefer M A, Chang J J, Hamner C and Engels P 2011 Phys. Rev. A $84041605(\mathrm{R})$.

[31] Hamner C, Zhang Y, Chang J J, Zhang C and Engels P 2013 Phys. Rev. Lett. 111264101 